\title{
Smart Television Services Using NFV/SDN Network Management
}

\author{
Nawar Jawad $^{\circledR}$, Mukhald Salih, Kareem Ali, Benjamin Meunier, Yue Zhang ${ }^{\circledR}$, Xun Zhang ${ }^{\circledR}$, Rudolf Zetik, \\ Charilaos Zarakovitis, Harilaos Koumaras, Michail-Alexandros Kourtis, Lina Shi ${ }^{\circledR}$, \\ Wojciech Mazurczyk, and John Cosmas, Member, IEEE,
}

\begin{abstract}
Integrating joint network function virtualization (NFV) and software-defined networks (SDNs) with digital televisions (TVs) into home environments, has the potential to provide smart TV services to users, and improve their quality of experience (QoE). In this regard, this paper focuses on one of the next generation services so-called follow me service (FMS). FMS is a service offered by $5 \mathrm{gNB}$ to user equipments (UEs) in indoor environments (e.g., home), it enables its clients to use their smart phones to select media content from content servers, then cast it on the nearest TV set (e.g., living room) and continue watching on the next TV set (e.g., kitchen) while moving around the indoor coverage area. FMS can be provisioned by utilizing UEs geolocation information and robust mechanisms for switching between multiple $5 \mathrm{G}$ radio access technologies (RATs), based on the intelligence of the SDN/NFV intelligent home IP gateway of the Internet of Radio Light (IoRL) project paradigm. In view that the actual IoRL system is at its early development stage, we step forward by using Mininet platform to integrate SDN/NFV virtualization into $5 \mathrm{G}$ multi-RAT scenario and provide performance monitoring with measurements for the identified service. Simulation results show the effectiveness of our proposal under various use case scenarios by means of minimizing the packet loss rate and improving QoE of the home users.
\end{abstract}

Index Terms-Software defined networks, network function virtualisation, quality of experience, Internet of radio light, intelligent home IP gateway.

Manuscript received October 26, 2018; revised January 18, 2019; accepted January 30,2019 . This work was supported by the European Union's Horizon 2020 Research Program through Internet of Radio-Light Project under Grant H2020-ICT 761992. Part of this paper have been published in the Proceedings of the IEEE BMSB 2018, Valencia, Spain. (Corresponding author: Nawar Jawad.)

N. Jawad, M. Salih, K. Ali, B. Meunier, and J. Cosmas are with the Department of Electronic and Computer Engineering, Brunel University, Uxbridge UB8 3PH, U.K. (e-mail: nawar.jawad@brunel.ac.uk).

Y. Zhang is with the Department of Engineering, University of Leicester, Leicester LE1 7RH, U.K.

X. Zhang and L. Shi are with the Department of Electronic and Computer Engineering, Institute Supérieur d'Electronique de Paris, 75006 Paris, France.

R. Zetik is with the Department Wireless Distribution Systems, Fraunhofer Institute for Integrated Circuits IIS, Fraunhofer-Gesellschaft zur Foerderung der Angewandten Forschung E.V., Erlangen, Germany.

C. Zarakovitis, H. Koumaras, and M.-A. Kourtis are with the National Centre for Scientific Research-Demokritos, Institute of Informatics and Telecommunications, 15310 Athens, Greece.

W. Mazurczyk is with the Institute of Telecommunications/Cybersecurity Division, Warsaw University of Technology, Warsaw, Poland.

Color versions of one or more of the figures in this paper are available online at http://ieeexplore.ieee.org.

Digital Object Identifier 10.1109/TBC.2019.2898159

\section{INTRODUCTION}

$\mathbf{T}$ HE DEMAND for mobile video streaming services has grown exponentially over the recent years. According to Cisco's survey [1], video content represented the $55 \%$ of the overall mobile data traffic in 2015, while given that there will be over 5.5 billion mobile users by 2020 (equal to about $70 \%$ of the world's population), it is expected to become more than three quarters of the total mobile traffic by 2020 [3]. Relevant research findings further highlight that the indoor percentage of data traffic usage represents between $70 \%$ to $90 \%$ of the overall data traffic, while cellular systems within indoor environments experience poor performance due to low signal reception and high spectrum congestion [4], [5]. Therefore, it becomes widely accepted that there is a need for new indoor solution to cope with this drastic data growth. The mainstream methodology to approach these issues relies on standard technologies, which may not provide satisfactory results, due to its reliance on scarce bandwidth and limited data rates.

On other hand, smart Televisions (TVs) are Internetconnected appliances that offer numerous online features, such as an on-demand video content, Internet-based services, etc. However, using traditional remote controllers for browsing the available media contents on smart TVs is not as convenient as using smartphones, since the latter rely on contemporary screen mirroring via casting mechanisms, which enhances the remote controlling experience. Yet, an issue arises when using smartphones as remote controllers, which is the requirement for fine tuning between the smartphone and the TV set prior to sharing media content. Most phone manufacturers have developed methods for screen mirroring between smartphones and smart TVs such as Mirror Share, File Share, HTC Connect, Miracast, etc. Unfortunately, these remote mirroring developments are numerous, plus, they do not share a common approach, which makes it difficult to evolve towards a holistic approach that can serve a wide range of different mirroring and sharing services. The viewpoint of this paper is that the aforementioned open issues in cellular indoor environment and smart TV services can be well-addressed by adopting a new indoor solution that provides higher data rates and offers new services to enable direct sharing capabilities to multiple smart TVs, using generic smartphones as smart remote controllers without the need for compatibility and prioriI pairing processes. The Internet of Radio Light (IoRL) project is an indoor solution that represents an evolutionary system 
architecture that utilizes new access technologies namely, millimeter Wave (mmWave), and Visible Light Communication (VLC) with Intelligent Home IP Gateway (IHIPGW) built based on a Software Defined Networks (SDN)/ Network Function Virtualisation (NFV) network paradigm. It is also compatible with existing system/standards, e.g., IEEE 802.11, 802.15, 3GPP [2].

Our intention is to exploit the intelligence offered by the synergy between SDN and NFV technologies, to design and develop new smart TV solution with improved Quality of Experience (QoE). This solution enables a user to search content on-demand via smartphone, select media content to be viewed on a TV and leave the rest to be taken care of by the intelligence of the IoRL network. The required task is to enable users to continue viewing media content while moving among multiple TVs within the home. This is accomplished by using a users' location information to allow the SDN to perform smart switching of packet flows to multiple nearby TV devices seamlessly. We call this service Follow Me Service (FMS). FMS introduces two main features. Firstly, it enables the registered User Equipment (UE) to enjoy watching video content on the nearest smart TVs, whilst having the flexibility to continue watching the content while moving around the coverage premises. This process is managed without going through pairing and restarting the videos each time they change their location around the house, which improves the QoE of the home users. Secondly, it enables the UE to perform Video Multicasting (MC) within the home area. Selecting the MC feature within the FMS, configures the network to forward the video stream to all the available TV sets within the home environment, thereby performing video multicasting. Note that FMS features do not require any other action from the UEs, except to keep carrying their smartphones while moving around the home area. Moreover, the use of virtualisation technology in the network infrastructure, facilitates incorporating proxy servers, location servers, location database, etc. in form of Virtualised Network Functions (VNFs), which leads to an increase in the agility and flexibility of service deployment, as well as reduced traffic delays and latency. The performed work introduces and implements a new video unicasting and multicasting service for indoor environments that relies on network infrastructure, rather end users' devices' capabilities, as implemented conventionally.

The rest of the paper is organized as follows: Section II briefly introduces the background information on proxies, SDN, and NFV. Section III highlights related research work, while Section IV outlines the IoRL system architecture. Section V present the location estimation procedure in IoRL system, Section VI explains the FMS service in detail, Section VII presents the proposed service operation, Section VIII presents performance evaluations and results, and finally Section IX concludes the paper, and highlight the future work.

\section{TECHNICAL OVERVIEW}

This section briefly describes the various technologies used as the main building blocks of the IoRL system namely, proxies, SDN, and NFV. FMS is utilizing of these technologies to offer its service to users within IoRL network paradigm.

\section{A. Proxies}

The concept of the Proxy in mobile networks is to intercept and split the connection between the UE and the external content server, therefore seamlessly becoming the content server to the UE and a client to the content server. One type of proxy server is the TCP split connection proxy, where this type of server creates two separate asynchronous connections on both sides of the server namely, (upstream) from the proxy server towards content server, and (downstream) from proxy server downwards the UE. This separation provides the network operator with flexibility in the traffic management to achieve an enhancement to the system performance.

Depending on the network deployment, and user requirements, the use of proxies becomes beneficial by reducing the Round-Trip Time (RTT) for the data request by creating two separate asynchronous sessions, enabling data buffering at the proxy. This kind of content management promotes higher downstream speed supported by the high bandwidth available for the UEs. The use of such proxies provides complete control of the whole transport segment between the client devices and the proxy [6], which enables fine tuning of the connection link. For example, connections between the TV set and the proxy can use advanced protocol features which cannot be easily deployed in a public network due to potential third-party interference. Therefore, specially customized data routing patterns and protocol sessions can be established for providing the FMS for the IoRL clients.

\section{B. Software-Defined Networking}

SDN technology is an emerging and innovative model for network representation. It facilitates the network management and enables programmatically efficient network configuration. By separating the control plane of the network from the underlying forwarding plane, it creates a logically centralized control view of the network. A typical representation of SDN architecture includes three layers: the application layer, the control layer and the infrastructure layer. The application layer contains typical network applications, such as firewalls and load balancers. The control layer represents the brain of the network and is implemented by a centralized SDN controller. The infrastructure layer consists of physical or virtual forwarding devices in the network. A well-defined interface (known as the southbound interface), such as the OpenFlow protocol, is used for the communication between the centralized controller and the switches of the infrastructure layer. OpenFlow protocol is used to instruct the forwarding devices with flow rules for dealing with an incoming packet, whether to forward it to a specific port, drop it, forward it to the controller, or rewrite its content, e.g., a header. This is performed by flow matching, where each flow matches a subset of the traffic and performs one or more of the actions mentioned above. A northbound interface, such as RESTful APIs, allow applications to communicate with the controller helping network administrators to programmatically shape traffic and deploy network services. 


\section{Network Functions Virtualisation}

NFV technology enables deploying a specific network function without the need to use dedicated hardware and proprietary software. Instead, it runs software applications on generic computer hardware. This technology facilitates developing services within network infrastructure flexibly, only requiring Commercial off the Shelf (COTS) hardware that consists of processing, computing, and storage resources. This allows to create networks that can accommodate diverse use cases on a common network infrastructure, where each use case requires certain services, and these services are realized by the combination of network resources [7].

\section{RELATED WORK}

Researchers in [8] predicted that, traffic offloading solutions that keeps minimal service disruption for UEs becomes a requirement for mobile networks, to resolve traffic congestions via smart traffic management and offer new business opportunities. In [9], researchers presented adaptive broadcast techniques over LTE sub-frames and Wi-Fi access points, to optimize network performance as well as improve UE satisfaction levels. They have shown that broadcast offloading enables serving a higher number of subscribers with higher satisfaction levels, along with power savings on UE side and on eNB side. On the other hand, performance enhancing proxies PEPs have been studied by many researchers for the last decade, to analyse their role in network performance. Xu et al. [6] indicated that proxies in mobile networks can enhance network performance in terms of object fetch time especially when fetching larger packets over links that suffer from latency and packet loss. Farkas et al. [10] showed that, deploying a split connection proxy at the SGW could to highly improve LTE network performance in terms of file downloading, Web browsing and video streaming applications.

Researchers also looked into the use of UE geolocation information in mobile networks due to its potential in improving various network services. In [11] UE's location-awareness in 5G networks considered as an emerging tool, which can be utilized by network operators and designers to enhance network planning and resource optimization, due to the location estimation accuracy. Liu et al. [12] draw the road map of using location information in mobile network from the second to the fourth generation, and they also investigated and proposed multi- Radio Access Technology (RAT) positioning architecture for $5 \mathrm{G}$. In this architecture the potential of $5 \mathrm{G}$ sub-meter precision location system is used to meet the needs in terms of coverage, time delay and positioning accuracy in 5G networks. Koivisto et al. [13] utilized Direction of Arrival (DoA) and Time of Arrival (ToA) estimation in Access Nodes (ANs) to achieve below one-meter accuracy UE tracking. They think accuracy can offer substantial benefits and opportunities for location-based services. Similarly, in [14] and [15], researchers showed a sub-meter range positioning accuracy is also achievable for outdoor environment in 5G, specifically for automotive applications, paving the road for new services on top of these networks by exploiting the positioning accuracy.

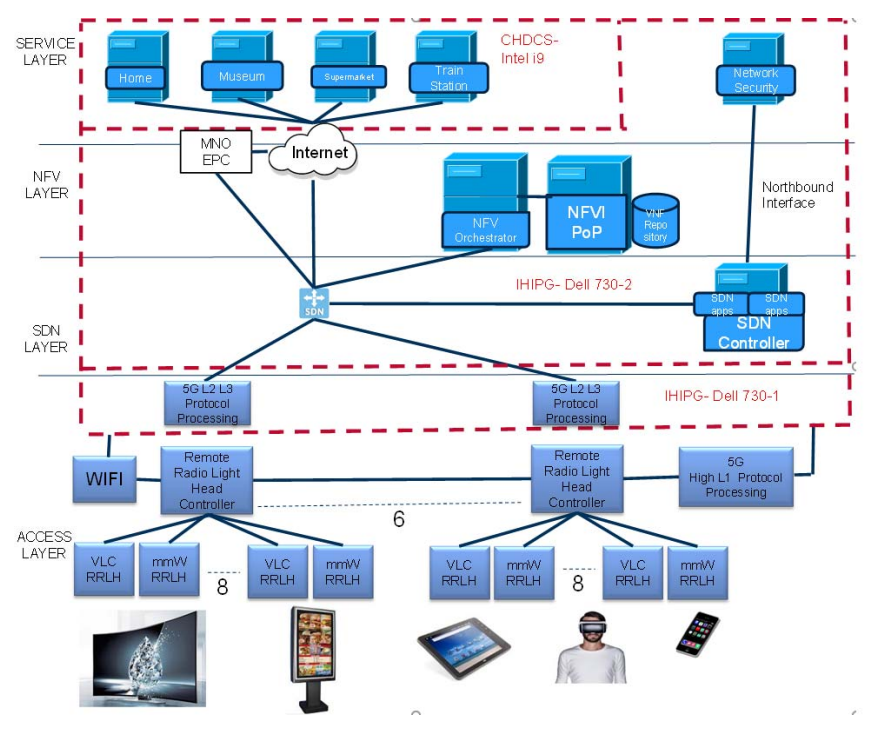

Fig. 1. IoRL Layered Architecture.

Meanwhile, SDN and NFV are considered by many researchers to be the enabler of the high flexibility and programmability of $5 \mathrm{G}$ networks. In this matter, Swetha and Raj [16] highlighted the need for optimized VNFs to maximize UEs' QoE when delivering high quality video content. While in [17], different types of caching techniques were presented, comparing various deployment locations within the network, where the authors emphasized on the role of NFV technology for the deployment of in-network caching to improve the performance of content delivery.

Based on the previous research findings mentioned earlier in this section, we proposed our novel service by combining the latest of all the previously mentioned technologies, to improve the UE's QoE.

\section{IORL SYSTEM ARCHITECTURE}

IoRL is a $5 \mathrm{G}$ gNB proposed as an indoor solution for improving the mobile networks indoor coverage as well as UE's QoE. IoRL architecture comprises of a radio access network, UE and IHIPGW. These are structured in four layers which are, the service layer, NFV layer, SDN layer, and the access layer. The system architecture is shown in Figure 1.

The IoRL approach includes designing and controlling a radio-light communication system that combines WLAN, mmWave and VLC access points, to offer access network for the users. At the same time IoRL enables deploying new services on top of its architecture. These services aim to improve UE's QoE by exploiting the SDN intelligence and NFV flexibility. OpenStack software is used to develop the virtualised SDN/NFV network architecture [18], [19], which enables flexible deployment of cloud computing for controlling large pools of compute, storage, network resources.

\section{A. IoRL Radio Access Network}

The access network represents the access layer of the IoRL architecture. It comprises of Remote Radio Light Head controllers (RRLH) installed through-out the indoor environment 


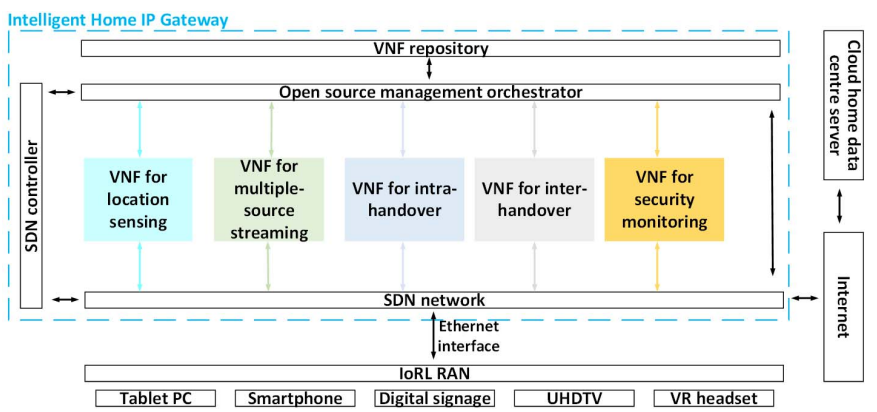

Fig. 2. Illustration of the IoRL Home Network with IHIPGW deployment of the identified VNF modeling.

premises. RRLH controllers contain mmWave and VLC modules together, and they are connected via 10Gbps Ethernet ring. Each RRLH Controller drives up to eight VLC and mmWave modules with the same transmission block sub-frame, in order to provide full coverage of the building premises.

\section{B. IoRL User Equipment UE}

User equipment (UE) integrates mmWave and VLC antennas within its design. mmWave antennas enable data duplex transmission, while VLC antennas enable data reception only. For FMS, the UE's smartphone acts as a remote controller, as well as a client location indicator as will be explained in FMS operation section later.

\section{Intelligent Home IP Gateway IHIPGW}

The IHIPGW hosts the intelligence of the IoRL system, since it is a contemporary SDN/NFV environment, which enables flexible and cost efficient deployment scheme. SDN offers a centralized control plane by logically separating the control plane from the underlying forwarding plane, which enables the IHIPGW to get a global view of the network, manage the routing of the data and enables services based on this data, as shown in Figure 2. The controller configures the forwarding device to route the traffic amongst multiple destinations that includes, IoRL Radio Access Network (RAN), Mobile Network Operator (MNO) Evolved Packet Core (EPC), local Internet breakout, or local applications running on multi-core Cloud Home Data Centre Server (CHDCS).

On the other hand, NFV facilitates the implementation of network functions as a software applications running on a nondedicated hardware in the form of VNF. This design increases system flexibility while reducing capital expenditure (CAPEX) and operational expenditure (OPEX). Meanwhile, the virtualised environment facilitates network service developers to create VNFs for location sensing, multiple-source streaming and security monitoring.

\section{LOCATION ESTIMATION}

In IoRL project, the system relies on VLC and mmWave positioning. It targets a high positioning accuracy of sub $10 \mathrm{~cm}$. This can be provided by combining both VLC and mmWave techniques.The main goal of position sensing in IoRL system is to support location based data access, monitoring, guiding and interactive applications. The main components of the location sensing architecture are location server, location database, location service client and RRLH controller.

The mmWave positioning system uses electromagnetic waves to determine the location of UEs. The RRLH controller is in charge of measuring time differences of arrival in the uplink. The mmWave measurements are reported by the RRLH controller to the radio resource controller, which communicates them to SDN in the form of packets using PDCP protocol. SDN transfers the mmWave measurements to the location database.

The positioning system based on VLC uses visible light signals for determining the positioning of UEs. The signals are transmitted by RRLH lamps and received by light sensors (e.g., photodiode or camera) on UEs. The VLC received signal strength data is measured in the downlink and transported to the location database by the UE's application software.

The location server estimates location coordinates of all connected UEs. The location estimation is based on the measured parameters as well as location assistance data. The location assistance data is represented by coordinates of all mmWave antennas and LEDs that are stored in the location database. The location server and the location database are VNFs implemented at IHIPGW. The location server is represented by location estimation and data fusion algorithms. The location estimation algorithms estimate UE postion based on the Time Differnt of Arrival (TDoA). The range differences $d i, 1(x, y, z)$ of the TDoA between the i-th and the first (reference) RRLH discribed in equation (1).

$$
\begin{aligned}
d i, 1(x, y, z)= & \sqrt{(x-x i)^{2}+(y-y i)^{2}+(z-z i)^{2}} \\
& -\sqrt{(x-x 1)^{2}+(y-y 1)^{2}+(z-z 1)^{2}} \\
& +(T i-T 1)
\end{aligned}
$$

where $x, y, z$ are the unknown coordinates of the UE, $x i, y i, z i$ are the coordinates of the i-th RRLH while $x 1, y 1, z 1$ are the coordinates of the reference RRLH and (Ti-Tl) represents the time offset between the it-th and the reference RRLHs. The solution of equation (1) is an example of least squares solution or iterative solution using linearization by Taylor series [20], [21]. The data fusion is represented by Kalman filter tracking algorithm which fuses the $\mathrm{mmW}$ and the VLC localization. The location database is implemented in MySQL, and it decouples UEs, RRLH controllers, location service clients and the location server. When RRLH controllers and UEs collect a new set of mmWave and VLC measurements they transfer them to the location database, then the location server obtains the measured parameters from the database together with the assistance data and does not need to interact with RRLH controllers and UEs. Estimated coordinates are saved in the corresponding table within the database.

The parameter measurements as well as the computation of UE coordinates are continuously performed by UEs, RRLH controllers and by the location server. The measured parameters as well as the location estimates are periodically updated in the location database. The location service clients access 
the location estimates only on demand according to the needs of their application software.

The mmWave based localization benefits from the large absolute bandwidth of license free 60GHz ISM frequency band and frequency bands that were deregulated for $5 \mathrm{G}$ communication systems such as $37-38.6 \mathrm{GHz}, 38.6-40$ or $64-71 \mathrm{GHz}$. The absolute bandwidth of mmWave ISM and $5 \mathrm{G}$ bands (with carrier aggregation) covers a couple of GHz. Such frequency bands can provide excellent time resolution which may result in sub-decimeter localization accuracy. This accuracy corresponds to the vision suggested for 5G networks [5], [22] and clearly outperforms accuracy of the commercial global navigation satellite system with its outdoor accuracy of about 5 meters, or the accuracy of indoor wireless local area network fingerprinting techniques which is about 34 meters.

The VLC based localization benefits from inexpensive installation by utilizing already existing illumination systems with few modifications. Its further advantage is its improved resistance to multipath in comparison to mmWave systems [23]. The accuracy corresponds to the lighting environment and LED device quality [24]. Based on three LED lights, the estimated UE position accuracy can be between (4-20) $\mathrm{cm}$ with different positioning algorithms [24]. We performed lab simulation to test our positioning system, where the lab dimensions were $5 \mathrm{~m}(\mathrm{~W}) * 5 \mathrm{~m}(\mathrm{~L}) * 3 \mathrm{~m}(\mathrm{H})$, and where it was equipped with 4 LED lights on the roof, which transmit visible light signals. The test subject moved in curve on the ground, ss can be seen in Figure 3 different position points. The VLC power received by the UE device can obtain an estimated distance between the UE and the LED. With the location of the LED, the estimated location of the UE can be calculated. $95 \%$ of the performed simulation tests showed position error within $10 \mathrm{~cm}$.

\section{Follow Me SERVice}

In this section, FMS architecture is presented by introducing all of the service building elements, as well as explaining the signaling sequence of the video content request.

\section{A. FMS Architecture}

System architecture of the FMS is shown in Figure 4. It consists of an end user application installed on a UE smart phone, SDN controller, Follow ME Application (FMA) written in python running on top of the SDN controller, and proxy/cache server. The main focus of FMA is to enable the SDN controller to update the Open virtual Switch (OvS) forwarding tables with the correct TV destination. FMA utilizes UE location update information, to ensure real time traffic switching instructions to be sent via SDN controller.

FMS seamlessly interacts with network entities to realize the service. The service procedure starts with UE registering for this service and requesting video content. Then UE chooses to either display the content on a specific TV set, or multicast it to all TV sets. FMA configures the OvS to forward the downlink stream to the correct TV device. The proxy server intercepts the TCP connection request originated by the UE,
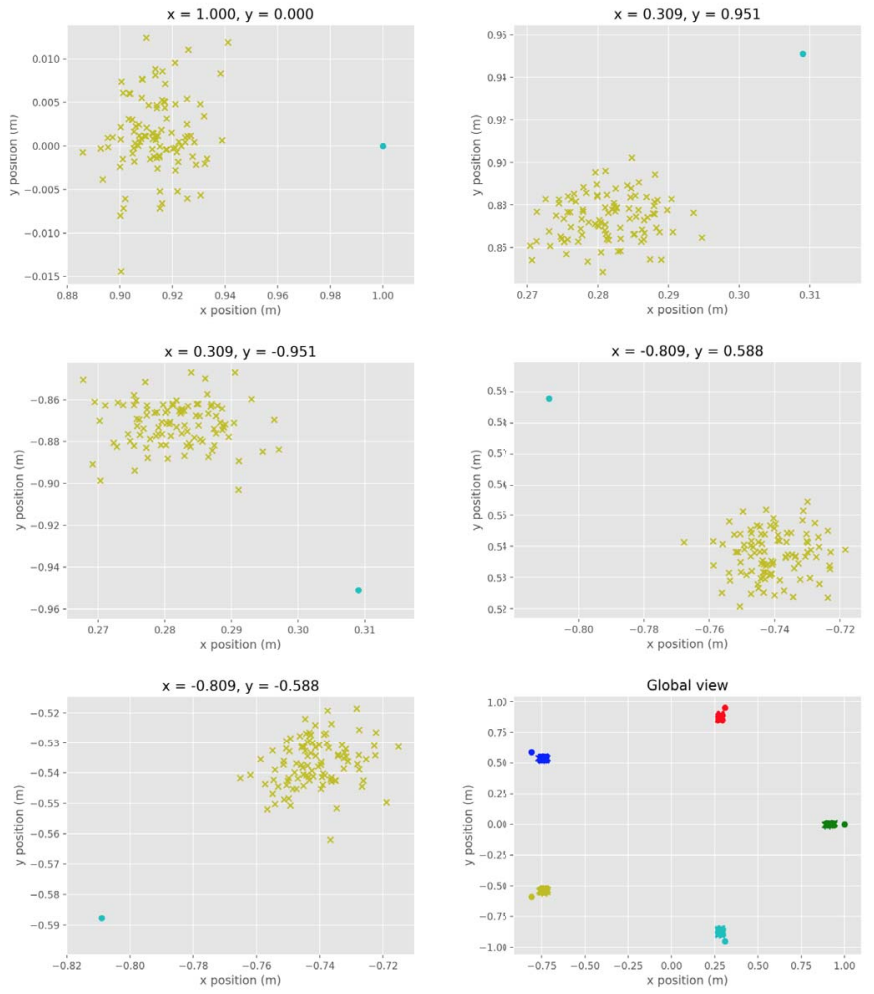

Fig. 3. VLC location estimation on different positions.

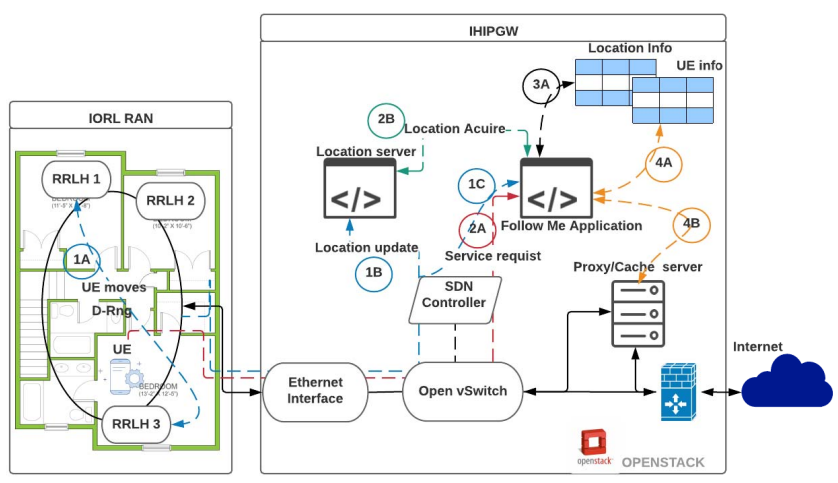

Fig. 4. FMS Architecture.

and fetches the video content from the content server, then passes the content to the end destination using UDP sessions.

TCP Proxy maximizes throughput by establishing asynchronous connection to the external server, downloads and buffers the downstream data at the proxy and generates ACKs towards the server. Then it uses connectionless UDP for sending the downstream to enable fast stream redirection. FMS exploits very wide bandwidth $(20-100 \mathrm{MHz})$ offered by IoRL, which highly reduces the probability of dropping packets due to congested network.

\section{B. FMS Signaling Sequence}

The connection establishment for the FMS and down streaming of the video is based on location updates of the UE, as shown in Figure 5. 


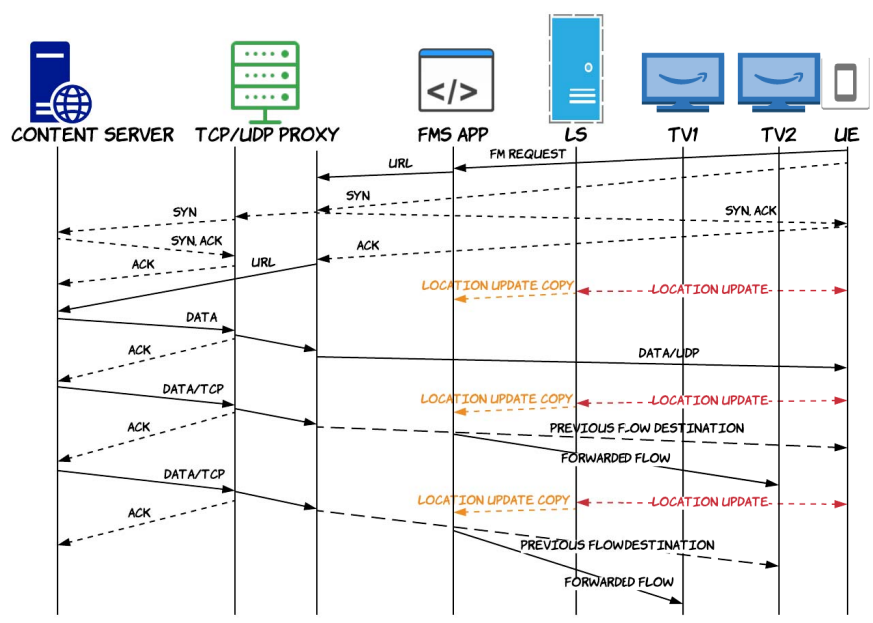

Fig. 5. FMS signaling sequence.

The request and response procedure shown in Figure 5 requires a client to request video content, consequently requested by the proxy from a content server (if it has not already been cached earlier). The proxy splits the session into two TCP sessions: i) UE-Proxy; ii) Proxy-Content Server. FMA monitors FMS clients' location updates, and modifies the OvS flow rules when necessary, ensuring traffic delivery to the correct TV destination. SDN intelligence plays an important role in improving FMS performance, where Ryu controller [25] configures the OvS to select the downstream path either via VLC/mmWave, or WiFi link, based on the acquired network metrics feedback.

\section{FMS OPERATION}

Follow Me Service uses three different components to provide its services to its clients within IoRL, namely, proxy server, FMA, and end user application. The service also utilizes the connection-less nature of UDP protocol to send the video to multiple destinations without the risk of losing datagrams due to hand-shakes and session establishments. In this section we introduce the operation procedure of the FMA, and the proxy server.

\section{A. FMA Operation Procedure}

As shown in Figure 6, FMA is registered as the listener for two types of incoming messages, follow me request messages, and location estimation messages.

1) When a location server sends a location estimation message to the location database, the FMA receives a copy of that message as shown in Figure 6. FMA checks if the UE is registered for the FMS by inspecting its local table of the registered UEs, and if a match is found, then it goes to step 3 (compare location information), otherwise, the message is ignored (UE is not registered with FMS).

2) If the received message is an initial request message from the UE, then FMA provides the controller with a forwarding policy to configure the OvS to forward the URL request to the proxy server. At the same time, the

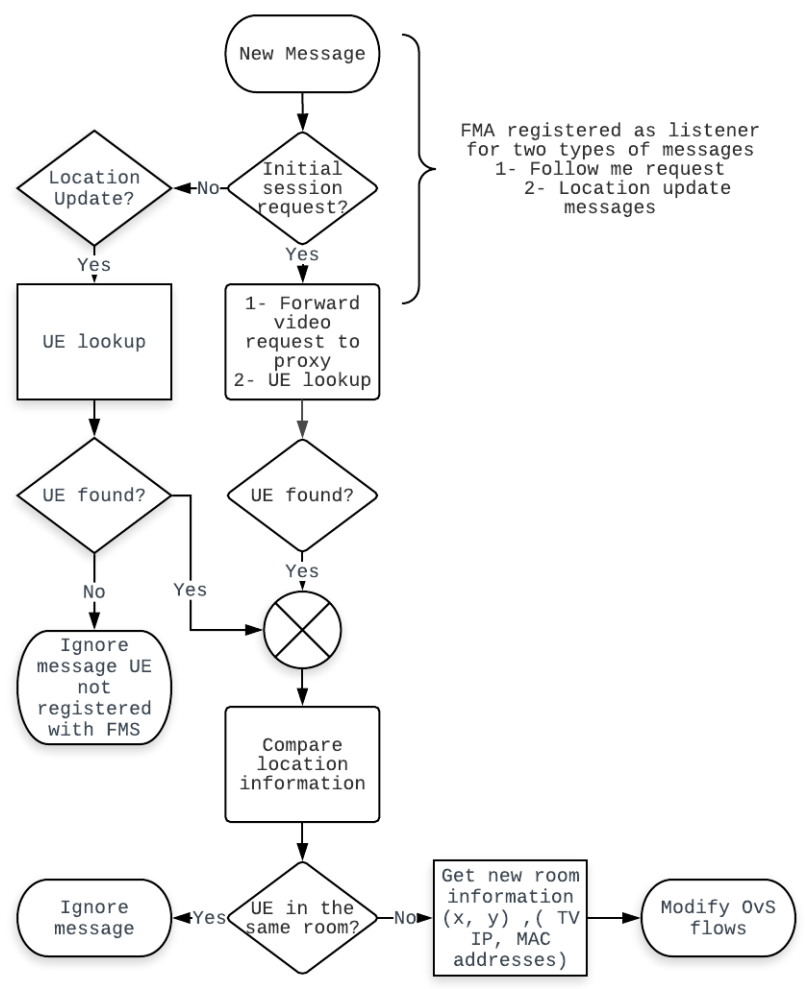

Fig. 6. FMA Flowchart.

FMA looks up its local table to check if the UE is a new client or if it is an existing client.

a) In case of a new client, FMA sends location acquire message to location database, and stores the UE location information from the returned response message.

b) Otherwise, it is an existing client, forwarding to step3 (compare location information).

3) FMA compares the UE current location information against the previously stored location information. Note that:

a) Client's location information is used by an algorithm to workout TV information (IP and MAC addresses) of the area where the client exists.

b) New client has no previous TV information; therefore, the current information is used by the OvS to forward the video to his first location.

4) If the client's new location is in the same room, then nothing needs to be done because the movement of the UEs does not require traffic redirection. While if the new location is in another room then the follow me procedure is triggered, which includes:

a) Storing the current TV information that corresponds to the client's current location.

b) Controller modifies the OvS flows to forward the flow to new location. If the UE is not in the FMS enabled area, then the controller sends a pause request message to the proxy server. 


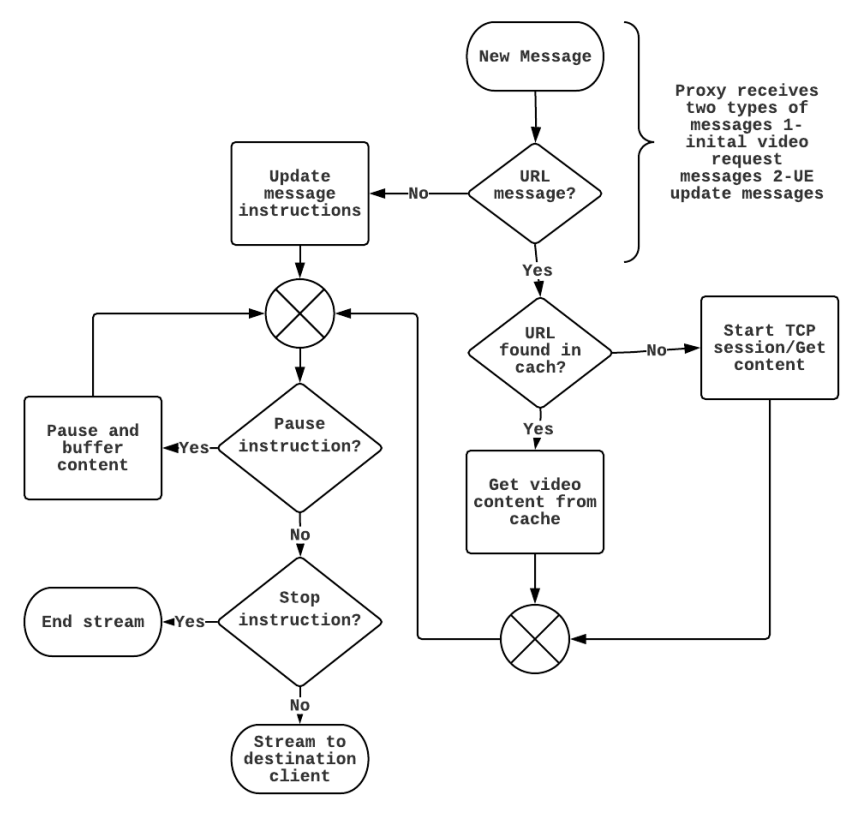

Fig. 7. Proxy Server Flowchart.

\section{B. Proxy Server Operation Procedure}

Proxy operation sequence is shown in Figure 7. When a new message is received by the proxy, it is either a client initial session request for a video or an update message from FMA.

1) In case of the received message is an initial session request

a) The proxy checks if the video is already available and cached on the local caching server, or it needs to request it from the external server.

2) In case of the received message is an update request message

a) If the update message includes a request to pause the video, then the proxy will pause and buffer the video until further request.

b) While if the update message includes a stop request, then the proxy will stop the video.

\section{ViII. Performance Evaluation and Results}

This section aims to quantify the potential of the proposed service, by providing multiple hypothetical scenarios in order to examine and evaluate the service performance. We first describe the used testbed and then present different performed scenarios.

\section{A. Testbed Description}

The test bed comprises of four layers, namely, service layer, control layer, access layer, and transport layer. Scenarios were implemented using Mininet emulation tool; Mininet was chosen since it is a well-known tool and has been widely used for emulating software defined networks. Our platform emulates two parts of the network, the IHIPGW, and the Radio Access Network (RAN). Since the actual RAN is under development, the implementation was simplified temporarily by assuming that layer2 of the RAN is capable of routing the packets to the correct RRLH controller. Then the RRLH controller forwards to the correct mmWave/ VLC modules, to be delivered to the correct TV set. These simplifications were made to show proof of concept of our developed service. The layers' details as the following:

- Service layer - this layer exposes the interaction between several network entities to provide the FMS. This service consumes UE location information, to perform video redirection to the current UE location.

- Control layer - it is the central part of the system architecture, it reflects the SDN intelligence by utilizing the UE location information to provide seamless traffic redirection by configuring the OvS to perform UDP traffic forwarding. This layer comprises of the Ryu SDN controller, as well as the SDN applications.

- Access layer - as previously mentioned, the RAN has not been completely implemented therefore the Mininet tool was used to emulate this layer.

- Transport layer - this layer allows interconnection between the access layer and service layer. It utilizes virtualised network entities to perform its task, such as, OvS, location database, and proxy server.

The deployed network topology in the hypothetical, multiple-room home scenarios is shown in Figure 8.

The network consists of one OvS, one Ryu controller, and eight hosts to emulate different network entities. Four of the eight hosts emulating TV sets are distributed within multiple rooms of the home. One host emulating the UE is registered as an FMS client, two hosts running in server-client mode are emulating the location server and location database. These servers are used to perform clients' location estimation and a storage mechanism. The last host is running Iperf traffic generator to emulate proxy server that is streaming cached video stream to a client. A Python script running as SDN application within the Ryu controller is used to configure the OvS by installing flows to route the video traffic to user's location. The controller is also configured to listen to the clients' location estimation update messages thereby, modifying the flows to reroute the video stream to the TV set within the new location of the client.

Several scenarios were used to demonstrate FMS reactions to client's movement within the home premises. The calculated quality of service (QoS) parameters in all scenarios were compared with each other to evaluate the system performance. Each test lasted 100(s), with Iperf generating 80 (Mbps) UDP stream, while UE location estimation data being randomly generated within home coordinates range and stored in the database.

1) Test One (No-Hop vs One-Hop Room Change Scenarios): In the first scenario run, a client is viewing a video stream on the nearest TV set. His location information values for the entire test period are confined to one room coordinates (Room1). Therefore, the video stream header contains the source IP address (10.0.0.7) of the proxy server, and destination socket address (10.0.0.4/4545), which remains unchanged. In the second scenario run, the client starts at one room receiving on socket address (10.0.0.4/4545) until he changes 


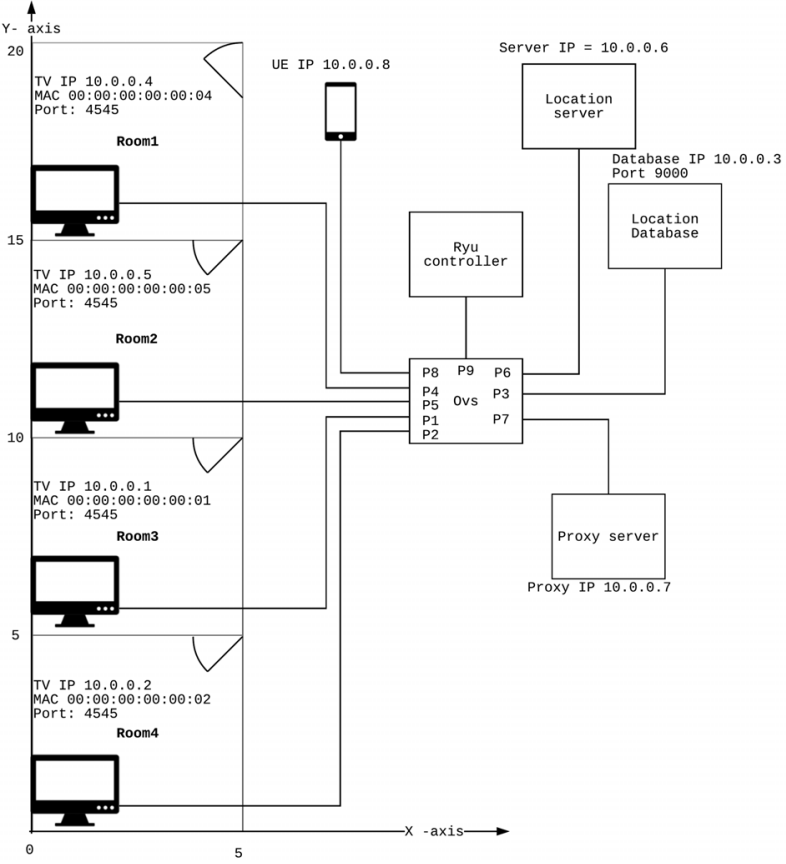

Fig. 8. Deployment scenario topology.

location to another room to continue viewing the video stream on the new socket address (10.0.0.5/4545). Some QoS parameters were measured (i.e., Throughput, packet loss, and Jitter) in each scenario and compared to each other to evaluate the system performance when the client's location change results in one-hop destination change. As shown in Figure 9, the stream jitter for the test scenarios were compared, where during the first scenario run, the SDN controller configured the OvS to route the stream to one destination address, while during the second scenario run the OvS was configured to reroute the stream twice during the test run time. Jitter ranged around $0.03(\mathrm{~ms})$ with some spikes in random times. Although the worst jitter experienced was below 0.1 (ms), this jitter was due to the nature of the Iperf tool. It is worth mentioning that, according to Cisco [26], the acceptable jitter for video is below $30(\mathrm{~ms})$, which proves that our system performance is excellent. System throughput for both scenarios of the first test were compared, as shown in Figure 10, where the throughput is ranging around $79.75(\mathrm{Mbps})$, with lowest throughput at 79.5 (Mbps); this result shows very good system performance during the first test scenarios.

2) Test Two (Two-Hop, Three-Hops, and Four-Hop Client Room Change Scenarios): In the first scenario run of this test, a client is requesting a video stream to be viewed on the TV set of Room1 (socket address10.0.0.4/4545), for $(35 \mathrm{~s})$, then changes his location to enter another room (Room 3) and continues watching the video stream for $(19 \mathrm{~s})$ on the new room's TV set (socket address 10.0.0.1/4545). The client finally renters the first room (Room1) and continues watching the rest of the video stream (46 s) on the room's TV set (socket address

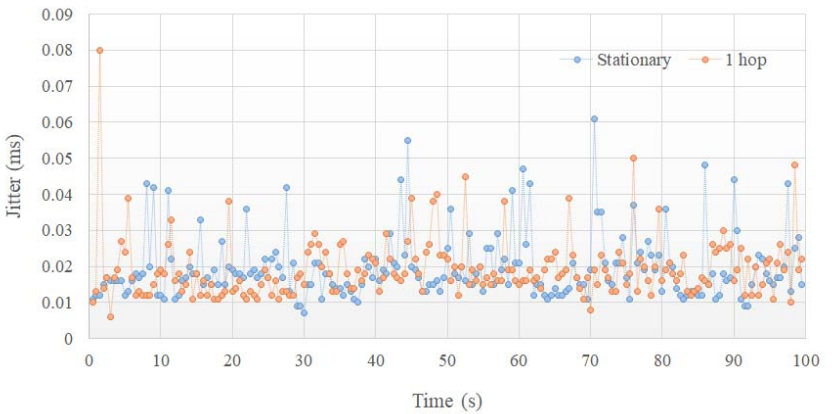

Fig. 9. Jitter No-hop vs one-hop scenarios.

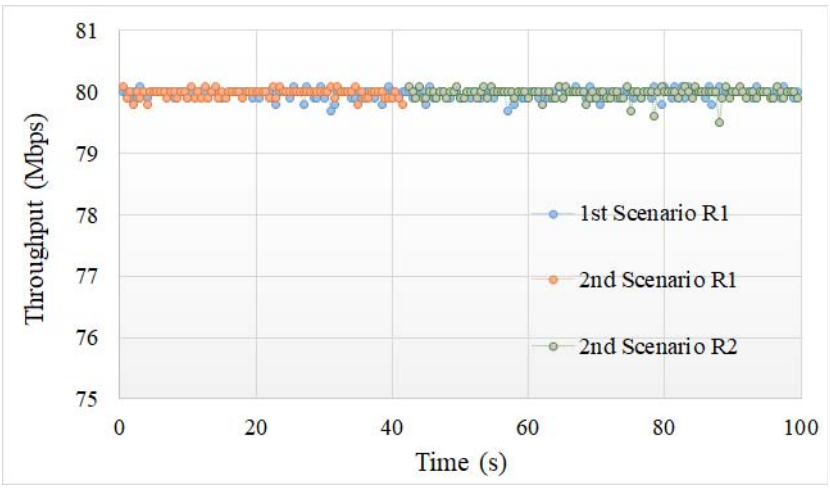

Fig. 10. Throughput No-hop vs one-hop scenario.

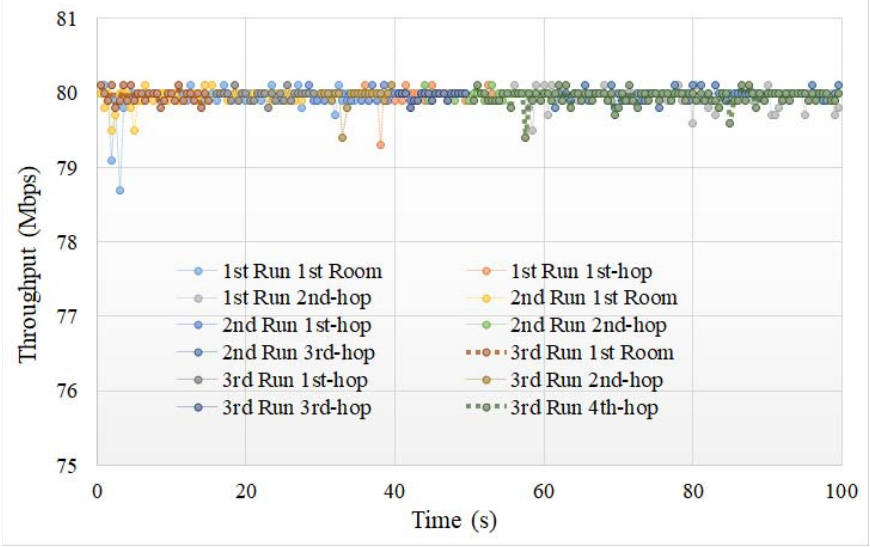

Fig. 11. Throughput two, three, and four hops.

10.0.0.1/4545). Similarly, in the second scenario of this test the client starts in one room viewing the video stream from the proxy server on the nearest TV set for (27.5 s). He then changes his location between rooms resulting in rerouting the video stream three times to the socket addresses (10.0.05/4545, 10.0.0.1/4545, and $10.0 .0 .2 / 4545)$ at times $(27.5,41.5$, and $59 \mathrm{~s})$ respectively, to accommodate continuous viewing of the video stream in all locations. Finally, the client in the third scenario has started viewing in one room on socket address (10.0.0.4/4545) and then changed rooms for three more times, resulting in changing destination socket addresses in the following pattern $(10.0 .05 / 4545,10.0 .0 .1 / 4545$, 


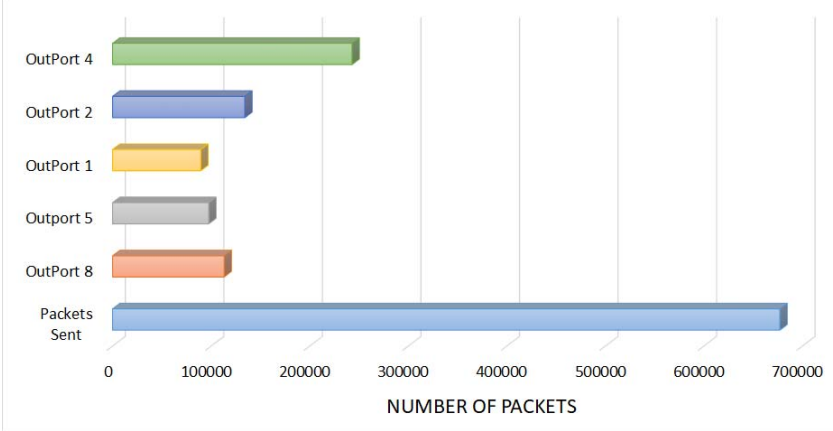

Fig. 12. Packet loss.

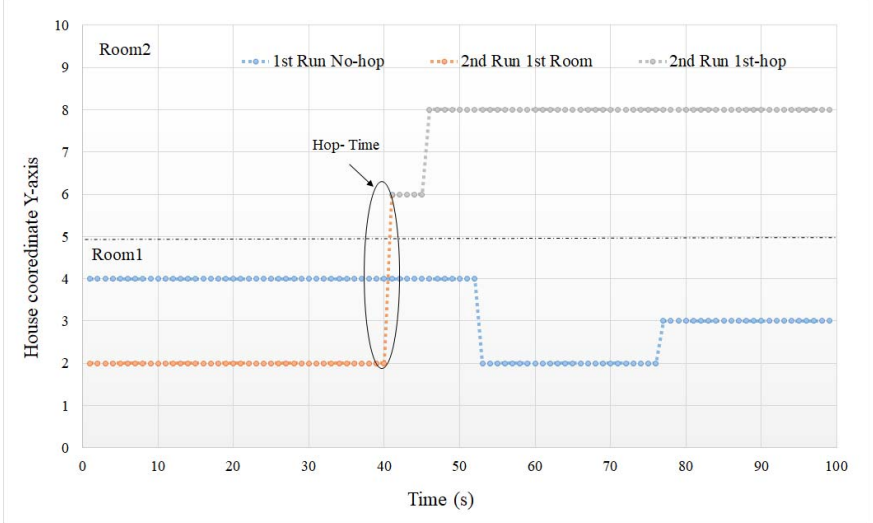

Fig. 13. Hop-time No-hop vs One-hop scenarios.

10.0.0.2/4545 and 10.0.0.1/4545) at times $(15,28.5,38.5$, and $48 \mathrm{~s}$ ) respectively. These frequent location changes enabled us to examine the system performance during and after these changing times to evaluate how it may or may not affect user experience. The system throughput among all three scenarios of the second test was compared, as can be seen in Figure 11, and it was observed that the throughput worst drop was below $78.75 \mathrm{Mbps}$ at one point during the first 5 seconds of the two-hop scenario, and it did not drop any further during the rest of the test run time. Furthermore, according to the test scenario, the first hop time of the two-hop scenario was at (35 s) of the run time, which proves that this drop was not related to service rerouting performance, rather to the Iperf traffic nature. Although $1 \%$ packet loss is considered acceptable according to [8], the systems performance showed resiliency to packet loss in the network, and no packet loss is encountered in the obtained results of different scenario runs. Figure 12 shows how the OvS distributes the received packets from the proxy server via different output ports based on the location of the UE. This proves that there was no packet loss within the network during testing. To stitch all results together, Figures 13 and 14 show the client's random location with respect to the hypothetical home, during test run time, time spent in each room for each scenario, and client's hop times. When comparing hop times for each run in each

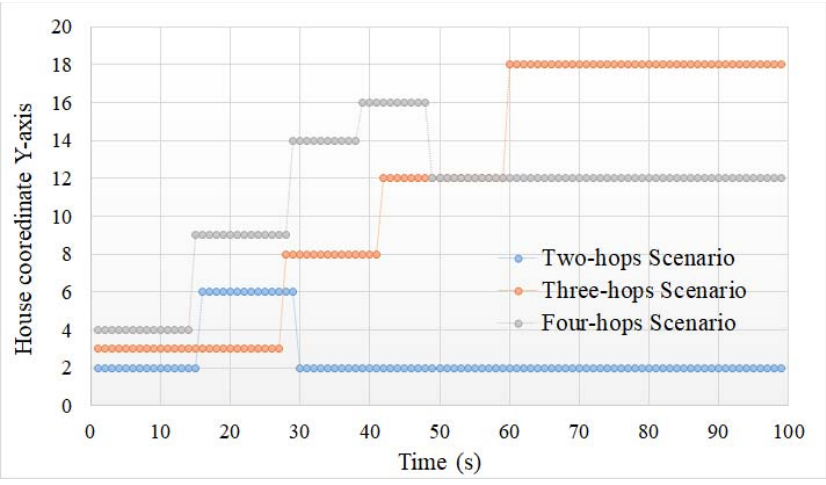

Fig. 14. Hop-time two, three, and four-hops scenarios.

scenario as shown in Figures 13, and 14 with system performance, it can be seen clearly that there is no performance degradation during handover times whatsoever. For instance at 41.5 (s), the client hands over from room 1 to room 2 at the one-hop scenario, and as can be seen in Figures 10 and 11 the jitter did not jump at this time or shortly after, furthermore the throughput of the system did not degrade at this time or shortly after.

\section{CONClusion And Future Work}

The paper presents a new service to improve QoE for IoRL clients, by using an SDN concept of configurable traffic routing reactively, and NFV technology for enabling flexible service deployment, as well as exploiting the huge bandwidth, and location estimation accuracy offered by the IoRL system. The objective of this paper is twofold. Firstly, it presents a new service for IoRL clients enabling them to continue watching a video stream of their choice on the nearest TV set of their current location within the home. Secondly, it proves the ability of the IoRL system to accommodate multiple services that provides higher $\mathrm{QoE}$ for its clients due to its flexible and intelligent design. The system testing showed high QoS performance parameters (zero packet loss due to route switching, very high throughput and $0.03 \mathrm{~ms}$ jitter). The current service performance tests were performed by emulating the RAN network since it is in the development stage, as well as assuming the video contents are existing in the local cache server. Future works may include implementing a TCP/UDP proxy server at the eNodeB edge to perform the TCP-UDP mapping of the video stream as well as designing a unified search platform for FMS clients, allowing for searching a media content from different media providers within one platform.

\section{REFERENCES}

[1] Cisco Visual Networking Index: Global Mobile Data Traffic Forecast Update, 2016-2021 White Paper, Cisco, San Jose, CA, USA. Accessed: Sep. 20, 2018. [Online]. Available: https://www.cisco.com/c/ en/us/solutions/collateral/service-provider/visual-networking-index-vni/ mobile-white-paper-c11-520862.html

[2] J. Cosmas et al., "A scalable and license free 5G Internet of radio light architecture for services in homes \& businesses," in Proc. IEEE Int. Symp. Broadband Multimedia Syst. Broadcast. (BMSB), Valencia, Spain, 2018, pp. 1-6. 
[3] M. Ur-Rehman and G. A. Safdar, LTE Communications and Networks: Femtocells and Antenna Design Challenges. Hoboken, NJ, USA: Wiley, 2018.

[4] C.-Y. Oh, M. Y. Chung, H. Choo, and T.-J. Lee, "Resource allocation with partitioning criterion for macro-femto overlay cellular networks with fractional frequency reuse," Wireless Pers. Commun., vol. 68, no. 2, pp. 417-432, Jan. 2013.

[5] R. E. Hattachi and J. Erfanian, 5G White Paper, Next Gener. Mobile Netw. Alliance, Frankfurt, Germany, 2015.

[6] $\mathrm{X} . \mathrm{Xu}$ et al., "Investigating transparent Web proxies in cellular networks," in Proc. Passive Active Meas., 2015, pp. 262-276.

[7] J. Ordonez-Lucena et al., "Network slicing for 5G with SDN/NFV: Concepts, architectures, and challenges," IEEE Commun. Mag., vol. 55, no. 5, pp. 80-87, May 2017.

[8] M. Amani, A. Aijaz, N. Uddin, and A. H. Aghvami, "On mobile data offloading policies in heterogeneous wireless networks," in Proc. IEEE 77th Veh. Technol. Conf. (VTC Spring), Dresden, Germany, 2013, pp. $1-5$.

[9] C. Singhal and S. De, "UE-TV: User-centric energy-efficient HDTV broadcast over LTE and Wi-Fi," IEEE Trans. Mobile Comput., to be published. doi: 10.1109/TMC.2018.2864567.

[10] V. Farkas, B. Héder, and S. Nováczki, "A split connection TCP proxy in LTE networks," in Information and Communication Technologies, R. Szabó and and A. Vidács, Eds. Berlin, Germany: Springer, 2012, pp. 263-274.

[11] R. Di Taranto et al., "Location-aware communications for 5G networks: How location information can improve scalability, latency, and robustness of 5G," IEEE Signal Process. Mag., vol. 31, no. 6, pp. 102-112, Nov. 2014.

[12] Y. Liu, X. Shi, S. He, and Z. Shi, "Prospective positioning architecture and technologies in 5G networks," IEEE Netw., vol. 31, no. 6, pp. 115-121, Nov./Dec. 2017.

[13] M. Koivisto et al., "High-efficiency device positioning and locationaware communications in dense 5G networks," IEEE Commun. Mag., vol. 55, no. 8, pp. 188-195, Aug. 2017.

[14] J. A. del Peral-Rosado, J. A. López-Salcedo, S. Kim, and G. SecoGranados, "Feasibility study of 5G-based localization for assisted driving," in Proc. Int. Conf. Localization GNSS (ICL-GNSS), Barcelona, Spain, 2016, pp. 1-6.

[15] M. Koivisto et al., "Continuous high-accuracy radio positioning of cars in ultra-dense 5G networks," in Proc. 13th Int. Wireless Commun. Mobile Comput. Conf. (IWCMC), Valencia, Spain, 2017, pp. 115-120.
[16] S. Swetha and D. Raj, "Optimized video content delivery over 5G networks," in Proc. 2nd Int. Conf. Commun. Electron. Syst. (ICCES), Coimbatore, India, 2017, pp. 1000-1002.

[17] X. Wang, M. Chen, T. Taleb, A. Ksentini, and V. C. M. Leung, "Cache in the air: Exploiting content caching and delivery techniques for 5G systems," IEEE Commun. Mag., vol. 52, no. 2, pp. 131-139, Feb. 2014.

[18] J. H. Cox et al., "Advancing software-defined networks: A survey," IEEE Access, vol. 5, pp. 25487-25526, 2017.

[19] V. Nguyen, A. Brunstrom, K. Grinnemo, and J. Taheri, "SDN/NFV-based mobile packet core network architectures: A survey," IEEE Commun. Surveys Tuts., vol. 19, no. 3, pp. 1567-1602, 3rd Quart., 2017.

[20] G. Shen, R. Zetik, and R. S. Thoma, "Performance comparison of TOA and TDOA based location estimation algorithms in LOS environment," in Proc. 5th Workshop Position. Navig. Commun., Hanover, Germany, 2008, pp. 71-78.

[21] R. M. Buehrer, H. Wymeersch, and R. M. Vaghefi, "Collaborative sensor network localization: Algorithms and practical issues," Proc. IEEE, vol. 106, no. 6, pp. 1089-1114, Jun. 2018.

[22] P. Zhang, J. Lu, Y. Wang, and Q. Wang, "Cooperative localization in 5G networks: A survey," ICT Exp., vol. 3, no. 1, pp. 27-32, Mar. 2017.

[23] A. Arafa, S. Dalmiya, R. Klukas, and J. F. Holzman, "Angle-of-arrival reception for optical wireless location technology," Opt. Exp., vol. 23, no. 6, pp. 7755-7766, 2015.

[24] C. Huang and X. Zhang, "Impact and feasibility of darklight LED on indoor visible light positioning system," in Proc. IEEE ICUWB, Salamanca, Spain, 2017, pp. 1-5.

[25] Component-Based Software Defined Networking Framework. Accessed: Jan. 15, 2019. [Online]. Available: https://osrg.github.io/ryu/

[26] C. S. Lewis and S. Pickavance, Selecting MPLS VPN Services. Indianapolis, IN, USA: Cisco, 2006.

Authors' photographs and biographies not available at the time of publication. 